E3S Web of Conferences 1, 05005 (2013)

DOI: $10.1051 / \mathrm{e} 3 \operatorname{sconf} / 20130105005$

(c) Owned by the authors, published by EDP Sciences, 2013

\title{
Development of a Novel Fiber Optic Sensor Combined with a Fluorescence Turn-on Probe for $\mathrm{Cu}$ (II) Detection
}

\author{
J. Ma ${ }^{1}$, Y. Chiniforooshan ${ }^{1}$, W. H. Hao ${ }^{2}$, W.J. Bock ${ }^{1}$, Z. Y. Wang ${ }^{2}$ and E. Dabek-Zlotorzynska ${ }^{2,3}$ \\ ${ }^{1}$ Centre de recherche en photonique, Département d'informatique et d'ingénierie, Université du Québec en Outaouais, P. \\ O. Box 1250, Hull Station, Gatineau, Québec J8X 3X7, CANADA; ma.jianjun@uqo.ca wojtek.bock@uqo.ca \\ ${ }^{2}$ Department of Chemistry, Carleton University, 1125 Colonel By Drive, Ottawa, Ontario, K1S 5B6, CANADA; \\ whao@connect.carleton.ca; wayne_wang@carleton.ca \\ ${ }^{3}$ Environment Canada, 335 River Road, Ottawa, Ontario, K1A 0H3, CANADA; ewa.dabek@ec.gc.ca
}

\begin{abstract}
Existing staining-based methodology for the detection of metal ions is not well suited for real-time or in situ use. This is a significant problem, given that these ions can have a considerable impact on both human health and the environment. Thus, there is growing interest and need for simple, rapid and in-situ monitoring techniques for the purpose of detecting various target analytes (e.g. heavy metals), which is of a significant importance in many fields ranging from environmental monitoring to the study of intracellular processes. Among various sensors developed, optical fiber-optic sensors (FOS), based on fluorescence, are one class of sensors that address this goal [1]. Optical fibers are ideal for environmental sensing applications because of their ability to transmit optical signals to and from the sensing region without the use of free-space optics. In this work, we present, for the first time, a simple FOS incorporating novel fluorescence turn-on mechanism [2] that could detect $\mathrm{Cu}$ (II) as low as $10^{-4} \mathrm{M}$. Traditionally, fluorescence quenching or "turn-off" was used to detect $\mathrm{Cu}$ (II) [3]. In recent years, fluorescence "turn-on" emerges as a preferable tool. The developed fiber-optic sensor has two fiber leads and one probe head. One fiber lead includes 6 fibers for $\mathrm{He}-\mathrm{Ne}$ laser excitation light delivery (e-fibers). Another fiber lead has one receiving fiber (r-fiber) connected to an Ocean Optics QE65000 scientific grade spectrometer, which is interrogated by a computer via USB connection. The SpectroSuite software is used to observe and to record all spectra. The probe head combines all fibers together to form a coaxial structure with the r-fiber placed in the center. The key component in the proposed fluorescent sensing system is a probe prepared by binding a receptor containing a zwitterionic chromophore (M1), through noncovalent interactions, to the fluorescent polymer (P1) resulting in quenching its emission. The sensing mechanism involves the release of P1 (a fluorescent indicator) from its non-fluorescent indicator/receptor (P1/M1) pair upon exposure to $\mathrm{Cu}$ (II). The zwitterionic chromophore (M1) was found to show high selectivity and sensitivity to $\mathrm{Cu}$ (II), making it an ideal receptor for the recognition of $\mathrm{Cu}$ (II) [4]. The optimum operating conditions and performance characteristics for the new sensor will be discussed.
\end{abstract}

Key words: fiber optic sensor, fluorescence turn-on probe, copper

\section{Acknowledgements}

The authors gratefully acknowledge support for this project from the Natural Sciences and Engineering Research Council of Canada, from the Canada Research Chairs Program, and from a NATO Linkage Grant.

\section{References}

Ma, J.; Chiniforooshan, Y.; Hao, W. H.; Bock, W. J.; Wang, Z. Y. "Easily fabricated, robust fiber-optic probe for weak fluorescence detection: modeling and initial experimental evaluation", Optics Express, 2012, 20, 4805-4811.

Ma, J.; Hao,W. H.; Chiniforooshan, Y.; Bock, W. J.; Wang, Z. Y. "Easily Fabricated, Robust Fiber-optic Probe for Weak Fluorescence Detection Part II: "Off-on" Titration of Trace Heavy Metal" (In preparation)

D. R. Shnek, D. W. Pack, F. H. Arnold, and D. Y. Sasaki, "Metal-induced dispersion of lipid aggregates: a simple, selective, and sensitive fluorescent metal ion sensor" Angew. Chem., Int. Ed. Engl., 1995, 34, 905-907; 
R. Krämer, "Fluorescent chemosensors for $\mathrm{Cu}^{2+}$ ions: fast, selective, and highly sensitive" Angew. Chem., Int. Ed., 1998, 37, 772-773;

A. Torrado, G. K. Walkup and B. Imperiali, "Exploiting polypeptide motifs for the design of selective $\mathrm{Cu}$ (II) ion chemosensors," J. Am. Chem. Soc., 1998, 120, 609-610;

P. Grandini, F. Mancin, P. Tecilla, P. Scrimin and U.
Tonellato, "Exploiting the self-assembly strategy for the design of selective $\mathrm{Cu}^{\mathrm{II}}$ ion chemosensors", Angew. Chem., Int. Ed., 1999, 38, 3061-3064.

Hao, W. H.; McBride, A.; McBride, S.; Wang, Z. Y. "Colorimetric and near-infrared fluorescence turnon molecular probe for selective detection of cysteine in human plasma", J. Mater.Chem., 2011, 21,1040 . 